\title{
Expression of leukemia inhibitory factor in the rat retina following acute ocular hypertension
}

\author{
QIANQIAN HU ${ }^{1,2^{*}}$, CHANGQUAN HUANG ${ }^{1,2^{*}}$, YAO WANG ${ }^{1,3}$ and RENYI WU ${ }^{1,2}$ \\ ${ }^{1}$ Department of Glaucoma, Xiamen University Affiliated Eye Center; ${ }^{2}$ Eye Institute of Xiamen University, \\ Fujian Provincial Key Laboratory of Ophthalmology and Visual Science, Xiamen, Fujian 361001; \\ ${ }^{3}$ Department of Ophthalmology, First Hospital of Xi'an, Shaanxi Institute of Ophthalmology, \\ Shaanxi Provincial Key Lab of Ophthalmology, Xi'an, Shaanxi 710002, P.R. China
}

Received September 5, 2014; Accepted May 27, 2015

DOI: $10.3892 / \mathrm{mmr} .2015 .4287$

\begin{abstract}
The aim of the present study was to investigate the expression of leukemia inhibitory factor (LIF) and its downstream signaling pathways in the rat retina following acute ocular hypertension. The intraocular pressure of the rats was elevated to $110 \mathrm{mmHg}$ for $1 \mathrm{~h}$ by infusing the anterior chamber with normal saline. The retinal tissues were obtained $12 \mathrm{~h}, 24 \mathrm{~h}$, and 2, 3 and 7 days after termination of the ocular hypertension. Hematoxylin and eosin and terminal deoxynucleotidyl transferase dUTP nick end labeling (TUNEL) staining were performed to assess the morphological changes and the apoptosis of retinal cells, respectively. Quantification of the retinal ganglion cells (RGCs) was performed using fluorogold retrograde (FG) staining. The expression levels of LIF, LIF receptor (LIFR), signal transducers and activators of transcription 3 (STAT3), phosphorylated STAT3 (P-STAT3), Akt, phosphorylated-Akt (P-Akt), extracellular signal-regulated kinase (ERK) and phosphorylated ERK (P-ERK) were determined at different time-points following acute ocular hypertension using western blot analysis. Reverse transcription-quantitative polymerase chain reaction was performned to detect the mRNA expression levels of LIF and LIFR. The results revealed that $12 \mathrm{~h}, 24 \mathrm{~h}, 2,3$ and 7 days after reperfusion, the thickness of the inner nuclear layer and the inner plexiform layer was decreased, with a significant reduction in the number of RGCs, as determined using TUNEL and FG staining. The expression levels of LIF and LIFR were increased following acute ocular hypertension. At
\end{abstract}

Correspondence to: Professor Renyi Wu, Department of Glaucoma, Xiamen University Affiliated Eye Center, 336 Xiahe Road, Xiamen, Fujian 361001, P.R. China

E-mail:wubasel@hotmail.com

*Contributed equally

Key words: leukemia inhibitory factor, leukemia inhibitory factor receptor, Janus kinase-signal transducers and activators of transcription, Akt, acute ocular hypertension
$12 \mathrm{~h}$ post-retinal reperfusion, the expression levels of P-STAT3 and P-Akt were significantly upregulated, while the expression of P-ERK was decreased. The changes in the expression levels of LIF and LIFR suggested that LIF may be important in the process of degeneration/protection following retinal ischemia induced by acute ocular hypertension, via activation of the Janus kinase/STAT and Akt signaling pathways.

\section{Introduction}

Glaucoma remains the leading cause of irreversible blindness globally, and the apoptosis and/or death of retinal ganglion cells (RGCs) induced by abnormally high intraocular pressure (IOP) is the predominant pathophysiological basis of glaucoma (1). Lowering the elevated IOP in the eyes is the only effective treatment for glaucoma at present, however, a substantial proportion of patients with glaucoma experience visual field deterioration even following a significant IOP reduction, indicating progression of the disease (2). Neuroprotection aiming to rescue RGCs from degeneration is, therefore, considered to be important in glaucoma treatment in addition to IOP reduction.

Leukemia inhibitory factor (LIF), a member of the interleukin (IL)- 6 family, has been revealed to be a potential neuroprotective cytokine (3). Members of the IL-6 family do not share sequence homology, however, they are able to activate the same receptor, glycoprotein 130 (gp130) (4). Binding of LIF to its low-affinity LIF receptor (LIFR), results in the tyrosine phosphorylation of LIF, followed by the formation of a heterodimer. The heterodimer binds to its high-affinity receptor gp130 and exerts a variety of physiological effects via the activation of certain signaling pathways, including the Janus kinase/signal transducers and activators of transcription (JAK/STAT) pathway and mitogen-activated protein kinase (MAPK) pathways, including the extracellular signal-regulated kinase (ERK) and/or phosphatidylinositol-3 kinase (PI3K) pathways $(5,6)$.

LIF has been reported to prevent the death of axotomized sensory neurons in the dorsal root ganglia and promote peripheral regeneration in rats $(7,8)$. A previous study also demonstrated that endogenous LIF has an axon protective capacity in acute experimental autoimmune encephalomyelitis in mice (9). 
In the eye, LIF protects photoreceptor cells against degeneration and extends the lifespan of photoreceptors following light-induced retinal damage $(10,11)$. LIF is also reported to promote the in vitro generation, survival and maturation of oligodendrocytes of the rat optic nerve (12). Furthermore, in a rat glaucoma model, upregulation in the gene expression of LIF is detected in early optic nerve head injury (13), suggesting its potential role in the pathophysiology of glaucoma. However, the potential neuroprotective effect of LIF on RGCs in glaucoma remains to be fully elucidated. In the present study, the expression levels of LIF, LIFR and the downstream signaling pathway of LIF (STAT3, Akt and ERK1/2) were investigated in the rat retina following acute IOP elevation.

\section{Materials and methods}

Establishment of a rat acute ocular hypertension model. All experimental procedures were performed in accordance with the ARVO Statement for the Use of Animals in Ophthalmic and Vision Research and the experimental protocol was approved by the experimental animal ethics committee of Xiamen University (Xiamen, China). A total of 60 Sprague-Dawley rats (200-250 g) were purchased from the Shanghai Laboratory Animal Center (Shanghai, China). The rats were maintained on a 12-h light-dark cycle and were dark-adapted for at least $2 \mathrm{~h}$ prior to experiments. The animals had ad libitum access to food (standard lab chow) and water. The rats were injected intraperitoneally with chloral hydrate (10 mg/100 g; Sinopharm Chemical Reagent Co., Ltd., Shanghai, China) to ensure the animals remained immobile. Pupil dilatation was achieved using $0.5 \%$ tropicamide (Alcon, Fort Worth, TX, USA). Following topical administration of $0.5 \%$ proparacaine (Alcon), the anterior chamber was cannulated with a 7-scalp acupuncture, which was connected to a container carrying $500 \mathrm{ml}$ sterile normal saline. The IOP was increased to $110 \mathrm{mmHg}$ by elevating the saline reservoir to $150 \mathrm{~cm}$ above the eye for $60 \mathrm{~min}$. The body temperature of the rat was maintained at $37^{\circ} \mathrm{C}$ with a blanket. The opposite eye of each animal served as the normal control. The animals were sacrificed intraperitoneally with chloral hydrate $(20 \mathrm{mg} / 100 \mathrm{~g})$ 12 h, 24 h, 2, 3 or 7 days ( $=10 /$ group) following termination of the increase in IOP.

In situ staining of apoptotic cells. The rats ( $\mathrm{n}=3$ /group) were anesthetized with an intraperitoneal injection of chloral hydrate $(10 \mathrm{mg} / 100 \mathrm{~g})$ and perfused intracardially with cold 4\% paraformaldehyde (Sinopharm Chemical Reagent Co. Ltd.) in $0.1 \mathrm{~mol} / \mathrm{l}$ phosphate-buffered saline (PBS; pH 7.4). The eyes were immediately enucleated and the globes were postfixed in $4 \%$ paraformaldehyde in $0.1 \mathrm{~mol} / \mathrm{l} \mathrm{PBS}$ for $2 \mathrm{~h}$ at $4^{\circ} \mathrm{C}$. The cornea and the lens of the eye were removed, and the remaining eye cups were placed in the same fixative overnight. Prior to embedding in paraffin (Beijing Solarbio Science \& Technology Co., Ltd., Beijing, China), the eye cups were immersed in 70\%, 95\% and 100\% ethyl alcohol in series, and then embedded with paraffin. Paraffin sections of 5- $\mu$ m thickness were then cut with a semi-motorized rotary microtome (RM2245; Leica, Germany). To assess the end-stage apoptosis of the tissue, in situ terminal deoxynucleotidyl transferase dUTP nick end labeling (TUNEL) was performed on the retinal tissues using an assay kit (DeadEnd Fluorometric TUNEL system G3250; Promega Corporation, Madison, WI, USA), according to the manufacturer's instructions. The cellular nuclei were stained with DAPI (Vector Laboratories, Inc., Burlingame, CA, USA), and the apoptotic cells were examined under a laser confocal microscope (Fluoview 1000; Olympus, Tokyo, Japan). The cellular nuclei and apoptotic cells were counted in three sections from each sample. As a positive control, sections were incubated in DNase I $(0.5 \mu \mathrm{g} /$ $\mathrm{ml}$ ) prior to addition of the equilibration buffer. As a negative control, distilled water was used instead of the TdT reaction mixture.

Quantification of $R G C$ s in rat retinal flat mounts. Retrograde staining of the RGCs of the two eyes was achieved by injecting a fluorescent dye into the superior colliculus bilaterally. The rats ( $n=3 /$ group) were placed in a stereotactic apparatus (RWD Life Science Co. Ltd., Shenzhen, China), following intraperitoneal injection of $10 \mathrm{mg} / 100 \mathrm{~g}$ chloral hydrate to ensure the animals remained immobile and the skin of the skull was incised. The brain surface was exposed by perforating the parietal bone with a dental drill to facilitate dye injection. Fluorogold (FG; Fluorochrome LLC, Denver, CO, USA) was injected $(4 \% ; 3.0 \mu \mathrm{l}$ each) at a point $5.00 \mathrm{~mm}$ caudal to the bregma and $1.00 \mathrm{~mm}$ lateral to the midline on the two sides, to a depth of $5.00 \mathrm{~mm}$ from the surface of the skull.

Subsequently, 10 days after the injection of FG into the superior colliculus, the eyes were enucleated following sacrifice of the animals with an overdose of intraperitoneal $10 \%$ chloral hydrate. The eyes were fixed in $4 \%$ paraformaldehyde in PBS for $1 \mathrm{~h}$ in the dark at $4^{\circ} \mathrm{C}$. The anterior segments were removed and the eye cups were fixed in $4 \%$ paraformaldehyde/PBS for $30 \mathrm{~min}$ in the same conditions. A total of four radial cuts were made in the periphery of the eye cup, and the retina was carefully separated from the retinal pigment epithelium. A small cut was also made in the peripheral corner of the superior retinal portion in order to correctly identify retinal orientation.

The retina were then flat mounted on a glass slide and preserved in the dark at $4^{\circ} \mathrm{C}$. The retinal flat mounts were viewed using a fluorescence microscope (Leica DM2500; Leica Microsystems GmbH, Wetzlar, Germany; magnification, x100). The FG-labeled RGCs were manually counted in a blinded-manner, by another investigator, in each quadrant at a distance of $1 \mathrm{~mm}$ radial from the optic nerve. The total quantity of RGCs per $\mathrm{mm}^{2}$ in all four quadrants were calculated. Cells with an irregular shape, intense dye staining, or a smaller or larger size than typical RGCs were considered to be non-RGC cells, including microglia.

RNA extraction and reverse transcription-quantitative polymerase chain reaction $(R T-q P C R)$. Total RNA was extracted from the retina using TRIzol reagent (Invitrogen Life Technologies, Carlsbad, CA, USA). Reverse transcription was performed using Oligo 18T primers and RT reagents (Takara Bio Inc., Shiga, Japan), according to the manufacturer's instructions. RT-qPCR was performed with mRNA-specific primers. The following primer sequences were used: LIF, forward 5'-tcaactggctcaactcaacg-3' and reverse 5'-aaaggtgggaaatcc 
gtcat-3'; and LIFR, forward 5'-gctgacttctcgacctccac-3' and reverse 5'-ccagttccagtggtgacctt-3'. The qPCR reactions were performed on the StepOne Plus real-time PCR system (Applied Biosystems, Foster City, CA, USA) with SYBR Premix Ex Taq (Takara Bio Inc.) at $95^{\circ} \mathrm{C}$ for $10 \mathrm{~min}$, followed by 40 cycles of $95^{\circ} \mathrm{C}$ for $10 \mathrm{sec}, 57^{\circ} \mathrm{C}$ for $30 \mathrm{sec}$ and $75^{\circ} \mathrm{C}$ for $10 \mathrm{sec}$. Melt curve analysis was performed immediately between $65^{\circ} \mathrm{C}$ and $95^{\circ} \mathrm{C}$. All reactions were performed in triplicate and average threshold cycle $(\mathrm{Ct})$ values $>35$ were considered to be negative.

Western blot analysis. The total protein from the retina at different time-points was extracted using cold radioimmunoprecipitation assay buffer (Beijing Solarbio Science \& Technology Co., Ltd.). The protein concentration was determined with a bicinchoninic acid protein assay (Pierce Biotechnology, Inc., Rockford, IL, USA). Equal quantities ( $3 \mathrm{mg} / \mathrm{ml} ; 10 \mu \mathrm{l}$ ) of proteins extracted from the lysates were subjected to electrophoresis on $8 \%$ or $9 \%$ SDS-PAGE and then electrophoretically transferred onto polyvinylidene difluoride membranes (EMD Millipore, Billerica, MA, USA). Following blocking for $1 \mathrm{~h}$ in $2 \%$ bovine serum albumin, the membranes were incubated with primary antibodies against LIF (1:200; rabbit polyclonal anti-rat; Santa Cruz Biotechnology, Inc. Santa Cruz, CA, USA), LIFR (1:200; rabbit polyclonal antirat; Santa Cruz Biotechnology, Inc.), STAT3 (1:200, rabbit polyclonal anti- rat; Santa Cruz Biotechnology, Inc.), P-STAT3 (1:200; rabbit polyclonal anti- rat; Santa Cruz Biotechnology, Inc.), AKT (1:2,000; rabbit polyclonal anti-rat; Cell Signaling Technology, Danvers, MA, USA), p-AKT (1:1,000; rabbit polyclonal anti-rat; Cell Signaling Technology), ERK (1:1,000; rabbit polyclonal anti-rat; Cell Signaling Technology), p-ERK (1;1,000; rabbit polyclonal anti-rat; Cell Signaling Technology) and $\beta$-actin (1:10,000; Sigma-Aldrich, St. Louis, MO, USA) overnight at $4^{\circ} \mathrm{C}$. Following three washes with Tris-buffered saline containing $0.05 \%$ Tween-20 for 10 min each, the membranes were incubated with horseradish peroxidase-conjugated goat anti-rabbit $\operatorname{IgG}(1: 10,000$; Bio-Rad Laboratories, Inc., Hercules, CA, USA) for $1 \mathrm{~h}$ at room temperature. The specific bands were visualized using enhanced chemiluminescence reagents (Lulong Inc., Xiamen, China) and recorded using a transilluminator (ChemiDoc XRS, Bio-Rad Laboratories, Inc.). The data were analyzed using Image J software (National Institutes of Health, Bethesda, MD, USA). All experiments were performed at least three times with similar results.

Statistical analysis. All data are expressed as the mean \pm standard error of the mean and were analyzed using one-way analysis of variance followed by Tukey's multiple comparison test with SPSS version 13.0 software (SPSS, Inc., Chicago, IL, USA). $\mathrm{P}<0.05$ was considered to indicate a statistically significant difference.

\section{Results}

Retinal damage is induced by acute ocular hypertension. To assess the changes in retinal histopathology following acute ocular hypertension, hematoxylin and eosin staining was performed on the tissues. At 12 h, 24 h, 2, 3 and 7 days post-reperfusion, the thickness of the inner nuclear layer (INL) and the inner plexiform layer (IPL) gradually decreased, compared with the normal retina. The cell arrangement of the RGCs and the INL was irregular, with a significant reduction in the number of RGCs (Fig. 1). At 7 days post-reperfusion, cytoplasmic vacuolation occurred in the RGCs and cells were absent in the IPL (Fig. 1).

Apoptosis of the retinal cells is induced by acute ocular hypertension. TUNEL staining of normal retinas and retinas at $12 \mathrm{~h}, 24 \mathrm{~h}, 2,3$ or 7 days post-reperfusion after 60 min acute ocular hypertension is shown in Fig. 2. TUNEL-positive cells were absent in the normal retina (Fig. 2A), but were observed in the outer nuclear layer (ONL) and the INL in the group subjected to acute ocular hypertension at $12 \mathrm{~h}$ post-reperfusion (Fig. 2B-F), and the number of apoptotic cells peaked $24 \mathrm{~h}$ after reperfusion.

RGC loss occurs following acute ocular hypertension. The results of the FG retrograde staining of RGCs are shown in Fig. 3. Following acute ocular hypertension, significant reductions in the numbers of RGCs were found at $24 \mathrm{~h}, 2,3$ and 7 days post-retinal reperfusion.

Expression levels of LIF and LIFR in the rat retina following acute ocular hypertension. Western blot analysis was performed to assess the protein levels of LIF and LIFR, and RT-qPCR was performed to determine the mRNA expression levels of LIF and LIFR following acute ocular hypertension in the rat retina (Fig. 4). The western blot analysis demonstrated that the protein expression of LIF was significantly increased at $12 \mathrm{~h}, 24 \mathrm{~h}, 2,3$ and 7 days, and peaked $12 \mathrm{~h}$ after the IOP increase was terminated (Fig. 4A and B). The protein level of LIFR was also significantly higher, compared with the normal control at $12 \mathrm{~h}$ post-retinal reperfusion, and peaking 3 days after reperfusion (Fig. 4C and D). The mRNA expression levels of LIF and LIFR revealed the same patterns as the protein expression of LIF and LIFR in the rat retina following acute ocular hypertension (Fig. 4E and F).

Assessment of the activation of STAT3, Akt and ERK in the rat retina. Activation of the downstream pathways of LIF were assessed by investigating the expression levels of the phosphorylated forms of STAT3, Akt and ERK (Fig. 5). The results revealed that, at $12 \mathrm{~h}$ post-retinal reperfusion, the levels of P-STAT3 were significantly higher than that in the normal retina, and the highest levels of P-STAT3 were observed $24 \mathrm{~h}$ after retinal reperfusion (Fig. 5A and B). Upregulation in the phosphorylation of Akt was also observed, which peaked $12 \mathrm{~h}$ after retinal reperfusion (Fig. 5A and C). In contrast to P-STAT3 and P-Akt, the level of P-ERK1/2 was reduced from $12 \mathrm{~h}$ after retinal reperfusion in the rat retina (Fig. 5A and D).

\section{Discussion}

Glaucoma is a group of neurodegenerative diseases characterized by structural damage to the optic nerve and visual field loss, which are induced by pathologically raised IOP, resulting in the death of RGCs. In previous decades, efforts have been made to establish a stable and replicable animal model of 


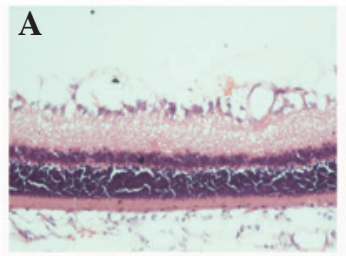

Normal

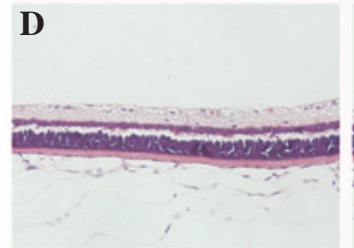

$2 \mathrm{~d}$

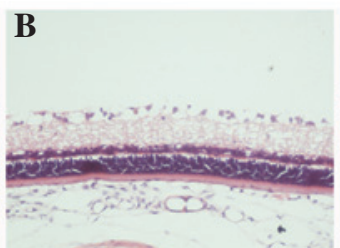

$12 \mathrm{~h}$

$\mathbf{E}$

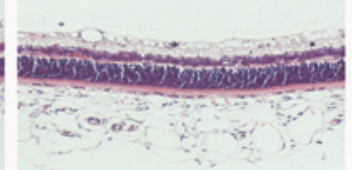

$3 \mathrm{~d}$

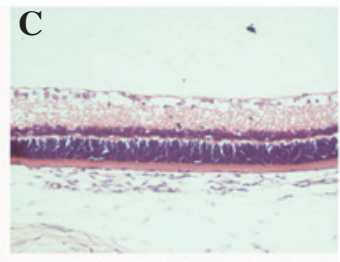

$24 \mathrm{~h}$

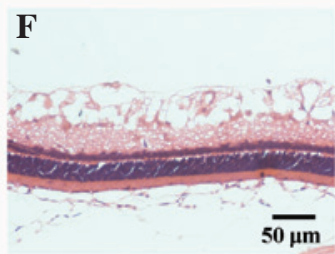

$7 \mathrm{~d}$

Figure 1. Hematoxylin and eosin staining of the rat retina following acute ocular hypertension for $1 \mathrm{~h}$. (A) Normal control; (B) $12 \mathrm{~h}$, (C) $24 \mathrm{~h}$, (D) 2 days, (E) 3 days and (F) 7 days after acute ocular hypertension.



Figure 2. TUNEL staining of rat retinal cells following acute ocular hypertension. (A) Normal control; (B) 12 h, (C) 24 h, (D) 2 days, (E) 3 days and (F) 7 days after acute ocular hypertension. No TUNEL-positive cells were observed in the normal control, however, (B-F) TUNEL-positive cells were present in the ONL and INL of the retina $12 \mathrm{~h}$ after acute ocular hypertension. Green indicates TUNEL-positive staining and blue indicates DAPI nuclear staining. ONL: outer nuclear layer; INL: inner nuclear layer; TUNEL, terminal deoxynucleotidyl transferase dUTP nick end labeling. glaucoma to investigate the pathology and treatment of the damaged nerve. By cannulating the anterior chamber with a needle connected to a reservoir containing sterile saline, the IOP of an animal can be raised to an abnormally high level and can be higher than the ocular perfusion pressure, preventing ocular blood flow and causing retinal ischemia, resulting in the death of RGCs. The apoptosis of RGCs induced by acute ocular hypertension is considered to simulate, at least partially, the pathophysiology of acute angle-closure glaucoma (14). In the present study, the results revealed thinning of the rat retina following acute ocular hypertension, particularly in the layers of the IPL, INL and RGCs. TUNEL-positive cell staining in the INL and ONL suggested the existence of cell apoptosis in the retina.

LIF is a multifunctional, pleiotropic cytokine (15) with a wide variety of physiological functions in the body, promoting the proliferation of primordial germ cells and spermatocyte differentiation $(16,17)$, inhibiting the differentiation of embryonic stem cells $(18,19)$ and being involved in bone formation (20,21). Furthermore, as a neurotrophic factor, LIF is reported to enhance the regeneration of axotomized sciatic nerves, accelerating the repair of the injured sensory nerve, promoting olfactory sensory neuron regeneration and corneal nerve recovery following laser in situ keratomileusis (22-25).

In the retina, LIF is produced predominantly in the Müller cells. Previous reports have demonstrated that the expression of LIF is markedly upregulated in the mouse retina when light damage is induced with bright cyclic light preconditioning $(26,27)$. The present study demonstrated that the number of apoptotic cells increased significantly $12 \mathrm{~h}$ after acute ocular hypertension and peaked at 24 , followed by a decrease. The apoptotic cells were located predominantly in the INL $12 \mathrm{~h}$ and $24 \mathrm{~h}$ after termination of ocular hypertension, while from day 2, the apoptotic cells were scattered in the ONL (Fig. 2). A significant reduction in the number of RGCs was noted at $24 \mathrm{~h}$ after acute ocular hypertension. The protein and mRNA expression levels of LIF were upregulated following acute ocular hypertension, peaking at $12 \mathrm{~h}$. The expression pattern of LIF was consistent with the change in the number of apoptotic cells in the retina (Fig. 4). The expression of LIFR was increased steadily following acute ocular 


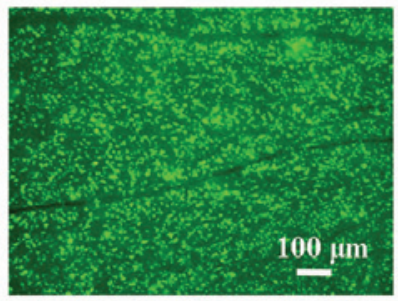

Normal

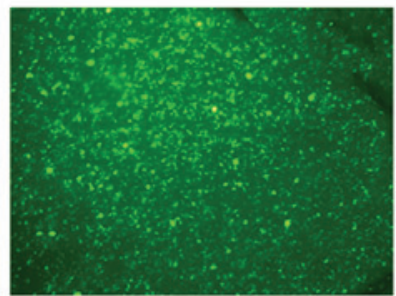

2d

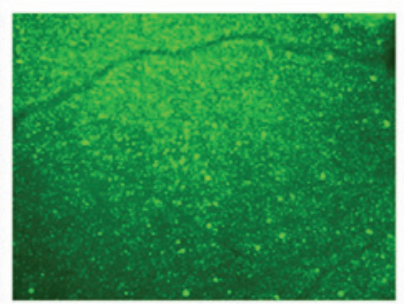

$12 \mathrm{~h}$

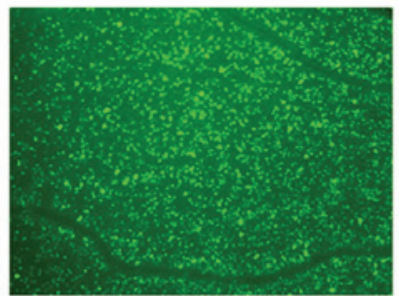

3d

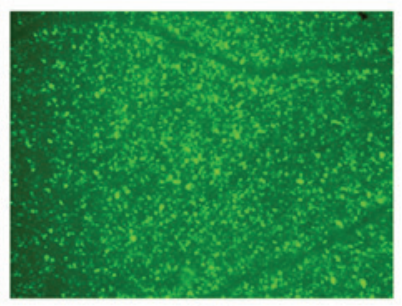

$24 \mathrm{~h}$

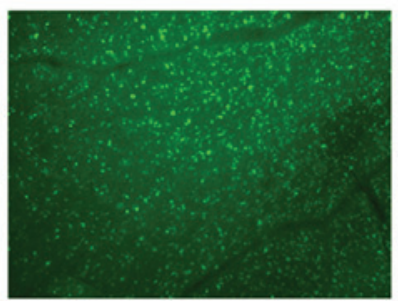

7d



Figure 3. Fluorogold retrograde staining of retinal ganglion cells in the rat retina following acute ocular hypertension. Data are expressed as the mean \pm standard error of the mean. ${ }^{* *} \mathrm{P}<0.01,{ }^{* * *} \mathrm{P}<0.001$, vs. normal. $\mathrm{N}$, normal control.

A
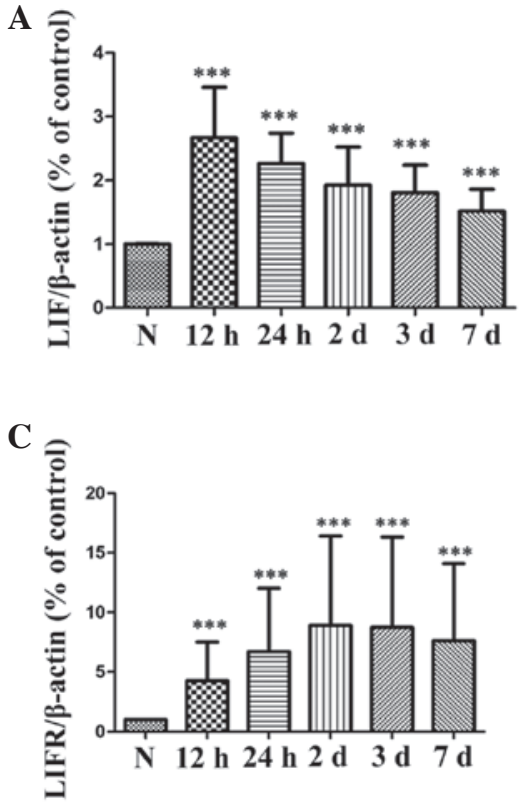

E

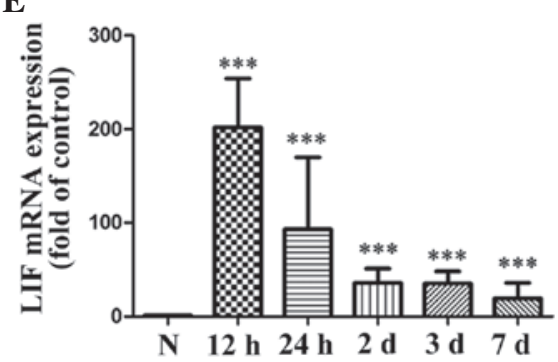

B

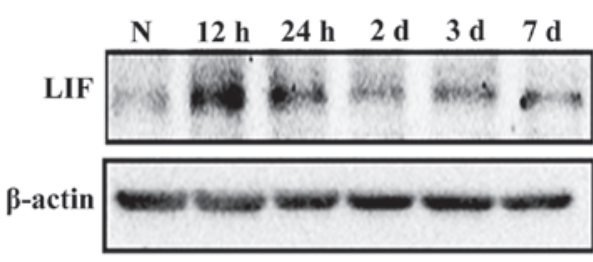

D

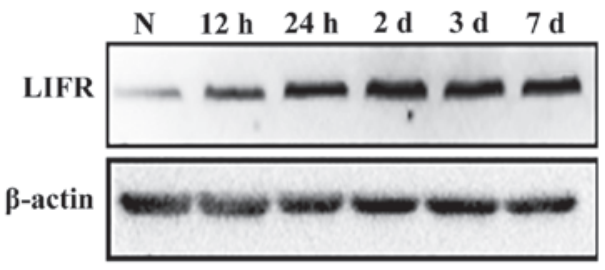

$\mathbf{F}$

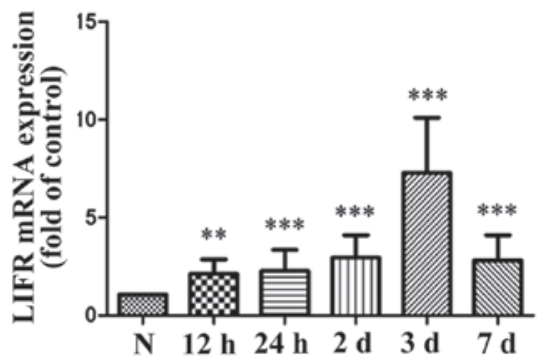

Figure 4. Expression levels of LIF and LIFR in the rat retina following acute ocular hypertension. Protein expression of (A and B) LIF and (C and D) LIFR was assessed by western blotting, and the mRNA levels of (E) LIF and (F) LIFR were tested by quantitative PCR assay. Data are expressed as the mean \pm standard error of the mean. ${ }^{* *} \mathrm{P}<0.01,{ }^{* * *} \mathrm{P}<0.001$, vs. $\mathrm{N}$ group. $\mathrm{N}$, normal control; LIF, leukemia inhibitory factor; LIFR, leukemia inhibitory factor receptor; PCR, polymerase chain reaction.

hypertension-induced ischemia and peaked after 3 days. An explanation for this inconsistency in the expression of LIFR and LIF was that the LIFR is able to bind with several cyto- kines in addition to LIF, including ciliary neurotrophic factor (CNTF), cardiotrophin-1 and oncostatin M, which, in turn alter the expression of LIFR (28-31). Notably, the expression 
A

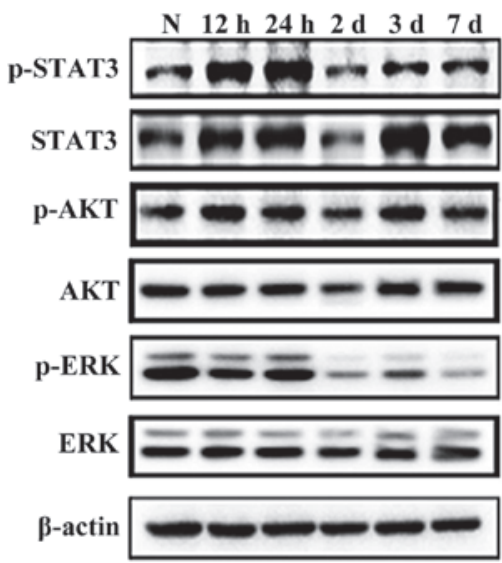

C

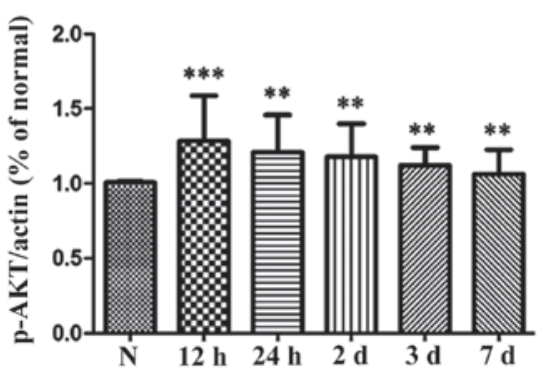

B

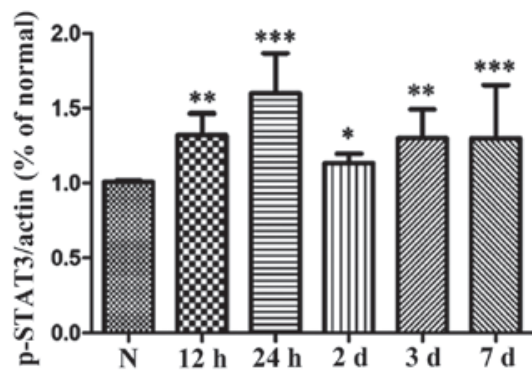

D

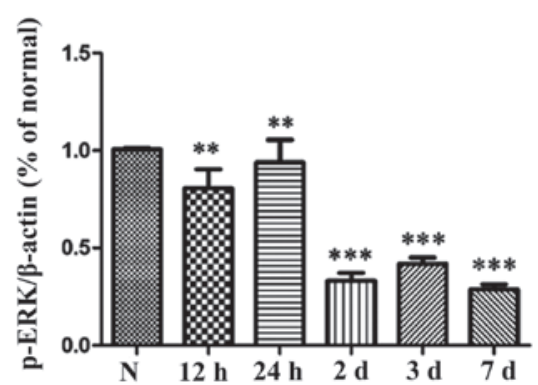

Figure 5. Expression levels of STAT3, Akt, ERK and their corresponding phosphorylated forms in the rat retina following acute ocular hypertension. The protein expression of STAT3, Akt, ERK and their corresponding phosphorylated forms was tested by (A) western blotting, and then (B-D) quantitative analysis, respectively. Data are expressed as the mean \pm standard error of the mean. ${ }^{*} \mathrm{P}<0.05,{ }^{* *} \mathrm{P}<0.01$ and ${ }^{* * *} \mathrm{P}<0.001$, vs. N group. N, normal control; STAT3, signal transducers and activators of transcription 3; ERK, extracellular signal-regulated kinase; p-, phosphorylated.

of CNTF has been reported to increase following acute ocular hypertension in the rat retina (32).

It has been reported that LIF is involved in the protection of photoreceptors against light-induced injury, predominantly through activation of the JAK-STAT3 pathway $(10,11)$. In the present study, the results of the western blot analysis demonstrated increased expression of P-STAT3 in the retina following acute ocular hypertension. In addition, the Akt pathway has been reported to have potent effects on neuroprotection in the retina. When phosphorylated, the Akt kinase promotes cell survival via the inactivation of apoptosis-associated components in the cell. Inactivation of the Akt signaling pathway is considered to be responsible for photoreceptor apoptosis in the mouse model of retinal degeneration (33). LIF has been reported to activate the PI3K signaling pathways, including Akt in cardiac myocytes $(3,6,34,35)$. In the present study, the increase in the expression of LIF was associated with elevated $\mathrm{P}-\mathrm{Akt}$ levels in the retina following retinal ischemia induced by acute ocular hypertension. These results suggested that LIF may mediate the processes of injury and repair in the retina through activation of the JAK-STAT3 and Akt signaling pathways.

As an MAPK pathway, the ERK pathway has been reported to be involved in cell proliferation and differentiation (36) when activated by mitogenic stimuli, including growth factors, cytokines, and phorbol esters. It has been reported that ERK activation is necessary for synaptic plasticity and memory in the central nervous system (37). In the rat retina, Roth et al (38) revealed that the expression of P-ERK peaked between 1 and $6 \mathrm{~h}$ following ischemia, followed by a decrease. Only weak expression levels of P-ERK were detected between days 3 and 7. The number of apoptotic cells in the RGC and photoreceptor layers are markedly decreased following the inhibition of ERK activation (38). In the present study, significant downregulation of the expression of P-ERK was observed in the rat retina following an acute increase in IOP. These observations were consistent with the findings of Roth et al (38), described above. Namura et al also demonstrated that intravenous injection of the MAPK/ERK kinase inhibitor, U0126, protects brain tissues from damage following forebrain and focal cerebral ischemia (39). However, the mechanisms responsible for cell death following ERK activation require further investigation.

In conclusion, the dynamic changes in the expression levels of LIF and LIFR observed in the present study suggested that LIF may be important in the process of degeneration/protection following acute ocular hypertension-induced retinal ischemia via the JAK-STAT3 and Akt signaling pathways.

\section{Acknowledgements}

The authors would like to thank Dr Zhen Zhang and Dr Yanfeng Chen for their assistance in animal experiments. This study was supported by the National Natural Science Foundation of China (grant no. 81170841) and Xiamen Science and Technology Project (grant nos. 3502Z20116011 and 3502Z20134040). 


\section{References}

1. Ji JZ, Elyaman W, Yip HK, Lee VW, Yick LW, Hugon J and So KF. CNTF promotes survival of retinal ganglion cells after induction of ocular hypertension in rats: The possible involvement of STAT3 pathway. Eur J nerurosci 19: 265-272, 2004.

2. Ciotu IM, Stoian I, Gaman L, Popescu MV and Atanasiu V: Biochemical changes and treatment in glaucoma. J Med Life 8: 28-31, 2015.

3. Heinrich PC, Behrmann I, Haan S, Hermanns HM, Müller-Newen G and Schaper F: Principles of interleukin (IL)-6-type cytokine signalling and its regulation. Biochem J 374: 1-20, 2003.

4. Yamauchi-Takihara K: Gp130-mediated pathway and let ventricular remodeling. J Card Fail 8 (6 Suppl): S374-S378, 2002.

5. Auernhammer CJ and Melmed S: Leukemia-inhibitory factor-neuroimmnue modulator of endocrine function. Endocr Rev 21: 313-345, 2000.

6. Oh H, Fujio Y, kunisada K, Hirota H, Matsui H, Kishimoto T and Yamauchi-Takihara K: Activation of phosphatidylinosito 3-kinase through glycoprotein 130 induces protein kinase B and p70 s6 kinase phosphorylation in cardiac myocytes. J Biol Chem 273: 9703-9710, 1998.

7. Cheema SS, Richards L, Murphy M and Bartlett PF: Leukemia inhibitory factor prevents the death of axotomised sensory neurons in the dorsal root ganglia of the neonatal rat. J Neurosci Res 37: 213-218, 1994

8. Mckay Hart A, Wiberg M and Terenghi G: Exogenous leukemia inhibitory factor enhances nerve regeneration after late secondary repair using a bioartificial nerve conduit. Br J plast surg 56: 444-450, 2003

9. Gresle MM, Alexandrou E, Wu Q, Egan G, Jokubaitis V, Ayers M, Jonas A, Doherty W, Friedhuber A, Shaw G, et al: Leukemia inhibitory factor protects axons in experimental autoimmune encephalomyelitis via an oligodendrocyte-independent mechanism. PLoS One 7: e47379, 2012.

10. Bürgi S, Samardziia M and Grimm C: Endogenous leukemia inhibitory factor protects photoreceptor cells against light-induced degeneration. Mol Vis 15: 1631-1637, 2009.

11. Joly S, Lange C, Thiersch M, Samardzija M and Grimm C: Leukemia inhibitory factor extends the lifespan of injured photoreceptors in vivo. J Neurosci 28: 13765-13774, 2008

12. Mayer M, Bhakoo K and Noble M: Ciliary nerrotrophic factor and leukemia inhibitory factor promote the generation, maturation and survival of oligodendrocytes in vitro. Development 120 : 143-153, 1994

13. Johnson EC, Doser TA, Cepurna WO, Dyck JA, Jia L, Guo Y, Lambert WS and Morrison JC: Cell proliferation and interleukin-6-type cytokine signaling are implicated by gene expression responses in early optic nerve head injury in rat glaucoma. Invest Ophthalmol Vis Sci 52: 504-518, 2011

14. Johnson TV and Tomarev SI: Rodent models of glaucoma. Brain Res Bull 81: 349-358, 2010.

15. Metcalf D: The unsolved enigmas of leukemia inhibitory factor. Stem Cells 21: 5-14, 2003.

16. Piguet-Pellorce C, Dorval-Coiffec I, Pham MD and Jégou B: Leukemia inhibitory factor expression and regulation within the testis. Endocrinonlogy 141: 1136-1141, 2000.

17. Sariola H: The neurotrophic factors in non-neuronal tissues. Cell Mol Life Sci 58: 1061-1066, 2001.

18. Williams RL, Hilton DJ, Pease S, Willson TA, Stewart CL, Gearing DP, Wagner EF, Metcalf D, Nicola NA and Gough NM: Myeloid leukemia inhibitory factor maintains the developmental potential of embryonic stem cells. Nature 336: 684-687, 1988.

19. Smith AG, Health JK, Donaldson DD, Wong GG, Moreau J, Stahl M and Rogers D: Inhibition of pluripotential embryonic stem cell differentiation by purified polypeptides. Nature 336 : 688-690, 1988.

20. Reid LR, Lowe C, Cornish J, Skinner SJ, Hilton DJ, Willson TA, Gearing DP and Martin TJ: Leukemia inhibitory factor: A nove bone-active cytokine. Endocrinology 126: 1416-1420, 1990.
21. Dazai S, Akita S, Hirano A, Rashid MA, Naito S, Akino K and Fujii T: Leukemia inhibitory factor enhances bone formation in calvarial bone defect. J Craniofac Srug 11: 513-520, 2000.

22. Dowsing BJ, Hayes A, Bennett TM, Morrison WA and Messina A: Effects of LIF dose and laminin plus fibronectin on axotomized sciatic nerves. Muscle Nerve 23: 1356-1364, 2000.

23. Cafferty WB, Gardiner NJ, Gavazzi I, Powell J, McMahon SB Heath JK, Munson J, Cohen J and Thompson SW: Leukemia inhibitory factor determines the growth status of injured adult sensory neurons. J Neurosci 21: 7161-7170, 2001.

24. Bauer S, Rasika S, Han J, Mauduit C, Raccurt M, Morel G, Jourdan F, Benahmed M, Moyse E and Patterson PH: Leukemia inhibitory factor is a key signal for injury-induced neurogenesis in the adult mouse olfactory epithelium. J Neurosci 23: 1792-1803, 2003

25. Pan S, Li L, Xu Z and Zhao J: Effect of leukemia inhibitory factor on corneal nerve regeneration of rabbit eyes after laser in situ keratomileusis. Neurosci Lett 499: 99-103, 2011.

26. Chollangi S, Wang J, Martin A, Quinn J and Ash JD: Preconditioning-induced protection from oxidative injury is mediated by leukemia inhibitory factor receptor (LIFR) and its ligands in the retina. Neurobiol Dis 34: 535-544, 2009.

27. Ueki Y, Le YZ, Chollangi S, Muller W and Ash JD Preconditioning-induced protection of photoreceptors requires activation of the signal-transducing receptor gp130 in photoreceptors. Proc Natl Acad Sci USA 106: 21389-21394, 2009.

28. Davis S, Aldrich TH, Stahl N, Pan L, Taga T, Kishimoto T, Ip NY and Yancopoulos GD: LIRR beta and gp130 as heterodimerizing signal transducers of the tripartite CNTF receptor. Science 260: 1805-1808, 1993

29. Pennica D, Shaw KJ, Swanson TA, Moore MW, Shelton DL, Zioncheck KA, Rosenthal A, Taga T, Paoni NF and Wood WI: Cardiotrophin-1. Biological activities and binding to the leukemia inhibitory factor receptor/gp130 signaling complex. J Biol Chem 270: 10915-10922, 1995.

30. Gearing DP and Bruce AG: Oncostatin M binds the high-affinity leukemia inhibitory factor receptor. New Biol 4: 61-65, 1992.

31. Plun-Favreau H, Perret D, Diveu C, Froger J, Chevalier S, Lelièvre E, Gascan $\mathrm{H}$ and Chabbert M: Leukemia inhibitory factor (LIF), cardiotrophin-1 and oncostatin M share structural binding determinants in the immunoglobulin-like domain of LIF receptor. J Biol Chem 278: 27169-27179, 2003.

32. Ju WK, Lee MY, Hofmann HD, Kirsch M, Oh SJ, Chung JW and Chun $\mathrm{MH}$ : Increased expression of ciliary neurotrophic factor receptor alpha mRNA in the ischemic rat retina. Neurosci Lett 283: 133-136, 2000.

33. Jomary C, Cullen J and Jones SE: Inactivation of the Akt survival pathway during photoreceptor apoptosis in the retinal degeneration mouse. Invest Ophthalmol Vis Sci 47: 1620-1629, 2006.

34. Hideshima T, Nakamura N, Chauhan D and Anderson KC: Biologic sequelae of interleukin-6 induced PI3-K/Akt signaling in multiple myeloma. Oncogene 20: 5991-6000, 2001.

35. Jee SH, Chiu HC, Tsai TF, Tsai WL, Liao YH, Chu CY and Kuo ML: The phosphotidyl inositol 3-kinase/Akt signal pathway is involved in interleukin-6-mediated Mcl-1 upregulation and anti-apoptosis activity in basal cell carcinoma cells. J Invest Dermatol 119: 1121-1127, 2002.

36. Chen J, Fujii K, Zhang L, Roberts T and Fu H: Raf-1 promotes cell survival by antagonizing apoptosis signal-regulating kinase 1 through a MEK-ERK independent mechanism. Proc Natl Acad Sci USA 98: 7783-7788, 2001.

37. Sweatt JD: The neuronal MAP kinase cascade: A biochemical signal integration system subserving synaptic plasticity and memory. J Neurochem 76: 1-10, 2001.

38. Roth S, Shaikh AR, Hennelly MM, Li Q, Bindokas V and Graham CE: Mitogen-activated protein kinases and retinal ischemia. Invest Ophthalmol Vis Sci 44: 5383-5395, 2003.

39. Namura S, Iihara K, Takami S, Nagata I, Kikuchi H, Matsushita K, Moskowitz MA, Bonventre JV and Alessandrini A: Intravenous administration of MEK inhibitor U0126 affords brain protection against forebrain ischemia and focal cerebral ischemia. Proc Natl Acad Sci USA 98: 11569-11574, 2001. 